Nat Biotechnol. 2008 December ; 26(12): 1348-1349. doi:10.1038/nbt1208-1348.

\title{
Cancer and complement
}

\author{
Suzanne Ostrand-Rosenberg \\ Department of Biological Sciences, University of Maryland Baltimore County, 1000 Hilltop Circle, \\ Baltimore, Maryland 21250, USA
}

\begin{abstract}
A component of the complement system promotes tumor growth by activating myeloid-derived suppressor cells.
\end{abstract}

The failure of cancer immunotherapies in clinical trials is due largely to tumor-induced immune suppression, mediated by cells such as myeloid-derived suppressor (MDS) cells and T regulatory cells. In a recent Nature Immunology article, Markiewski et al. ${ }^{1}$ elegantly demonstrate that MDS cells are recruited to tumors by the protein C5a, a component of the classical complement cascade. This study reveals yet another connection between inflammation and cancer and points to C5a as an attractive drug target for fighting cancer by suppressing the MDS cell population, possibly in conjunction with immunotherapeutics.

Complement is a collection of serum proteins that are integral to inflammatory processes and to innate immune responses to infection. C5a, also known as anaphylatoxin, is generated from C5 by C5 convertase, which includes C3a, a cleavage product of C3 (Fig. 1). Both C5a and $\mathrm{C} 3 \mathrm{a}$ are chemoattractants and pro-inflammatory mediators. Based on epidemiological and experimental data linking chronic inflammation with tumor onset and progression ${ }^{2}$, Markiewski et al. ${ }^{1}$ hypothesized that the inflammatory effects of complement may actually protect established tumors and enhance their growth. Using a murine model of cervical cancer and mice deficient in various complement components, they confirmed this hypothesis, showing that C5a deposited in the tumor vasculature attracts MDS cells, which express receptors for $\mathrm{C} 5 \mathrm{a}$, and boosts the potency of these cells by increasing their content of reactive oxygen and nitrogen species and of arginase-all of which are documented to contribute to MDS cell-mediated immunosuppression. Moreover, a peptide antagonist of the C5a receptor enhanced CD8+ T-cell anti-tumor responses and was as effective as the chemotherapeutic paclitaxel (Taxol) in retarding tumor growth.

Although MDS cells were originally observed in cancer patients and experimental animals $>30$ years ago, their role as spoilers of anti-tumor immunity is only now being appreciated. A heterogenous population of normal myeloid cells trapped in intermediate stages of differentiation, MDS cells accumulate in the blood, lymph nodes and at tumor sites in virtually all cancer patients. In healthy individuals, these cells differentiate into macrophages, dendritic cells and neutrophils, but tumors secrete a range of factors that disrupt normal differentiation of immune progenitor cells. MDS cells promote tumor growth by preventing the activation of $\mathrm{CD}^{+}$and $\mathrm{CD} 8^{+} \mathrm{T}$ lymphocytes, inhibiting natural-killer-cell cytotoxicity, stimulating tumorigenic cytokine production and increasing angio-genesis. Because MDS cells promote tumor growth through so many mechanisms, their elimination or inactivation should remove numerous barriers that interfere with cancer immunotherapies. 
The idea that chronic inflammation promotes tumor onset and progression was first suggested in the mid 1800s by the German pathologist Rudolf Virchow. Early hints that MDS cells might be involved included the observation that vascular endothelial growth factor (VEGF), a constituent of the pro-inflammatory milieu, induces the accumulation of MDS cells ${ }^{3}$ and is produced by these cells 4,5 . Recent studies directly linked inflammation and MDS cells by demonstrating that key pro-inflammatory mediators, including interleukins $1 \beta$ and 6 (IL-1 $\beta$, IL-6) ${ }^{6}, 7$, prostaglandin $\mathrm{E} 2\left(\mathrm{PGE}_{2}\right)^{8,9}$, and $\mathrm{S} 100 \mathrm{~A} 8 / \mathrm{A} 9$ proteins ${ }^{10,11}$ regulate the accumulation and suppressive activity of MDS cells. Like VEGF, the S100A8/A9 proteins are synthesized by MDS cells, thereby establishing an autocrine feedback loop that maintains MDS cell levels (Fig. 1).

Nevertheless, the finding by Markiewski et al. ${ }^{1}$ that the complement component C5a promotes tumor growth is unexpected, as complement-dependent cytotoxicity, arising from the binding of antibodies to complement, has historically been thought to contribute to the destruction of cancer cells. Although the discovery that malignant cells express regulatory proteins that protect them from complement-mediated lysis ${ }^{12}$ dashed hopes for antibody therapy based on this mechanism, until now there has been no evidence that complement actually facilitates tumor progression.

The results of Markiewski et al. ${ }^{1}$ further solidify the role of chronic inflammation in cancer and add new candidate drug targets for blocking MDS cell-mediated immunosuppression. Heterogeneity in the MDS cell phenotypes of individuals with histologically distinct tumors presumably reflects differences in the repertoires of MDS cell-inducing factors produced by cancer cells and complicates prospects for eliminating these cells. Different pro-inflammatory factors may have to be targeted in different patients, requiring drugs against a wide range of proteins that restrict immunosuppression. Moreover, Markiewski et al. ${ }^{1}$ report that, in the blood and spleens of tumor-bearing mice, both neutrophil-like and monocyte-like MDS cells express cell-surface C5a receptors, whereas in tumors, only neutro-phil-like MDS cells have these receptors. This suggests heterogeneity of MDS cells within a single individual, again emphasizing the need for multiple anti-inflammatory agents.

For individuals with established cancers, it is well accepted that MDS cells are immunosuppressive and that eliminating this cell population should increase the efficacy of cancer immunotherapies and vaccines that rely on active immunization. It has also been proposed that, before solid tumors become apparent, MDS cells facilitate the outgrowth of transformed cells by blocking the immune surveil-lance that would otherwise eliminate them $^{7}$. This hypothesis is supported by the finding of reduced tumor incidence in individuals who do not have cancer but have received long-term treatment with inhibitors of $\mathrm{PGE}_{2}$ or with nonsteroidal anti-inflammatory drugs such as aspirin. If the hypothesis is correct, drugs targeting pro-inflammatory mediators such as $\mathrm{C} 5 \mathrm{a}, \mathrm{IL}-1 \beta, \mathrm{IL}-6, \mathrm{PGE}_{2}$ and S100A8/A9-all of which activate MDS cells - may also be useful prophylactic agents. As relatively few side effects are reported for complement-directed therapy ${ }^{1}$, repurposing C5a inhibitors may be an attractive option. Regardless of the outcome of further investigations, MDS cells are undoubtedly among the most potent inhibitors of anti-tumor immunity, and the work of Markiewski et al. ${ }^{1}$ offers a promising avenue for eliminating these detrimental cells.

\section{References}

1. Markiewski MM, et al. Nat. Immunol 2008;9:1225-1235. [PubMed: 18820683]

2. Coussens LM, Werb Z. Nature 2002;420:860-867. [PubMed: 12490959]

3. Gabrilovich DI, et al. Nat. Med 1996;2:1096-1103. [PubMed: 8837607]

4. Yang L, et al. Cancer Cell 2004;6:409-421. [PubMed: 15488763]

5. Melani C, Chiodoni C, Forni G, Colombo MP. Blood 2003;102:2138-2145. [PubMed: 12750171] 
6. Song X, et al. J. Immunol 2005;175:8200-8208. [PubMed: 16339559]

7. Bunt SK, et al. J. Immunol 2006;176:284-290. [PubMed: 16365420]

8. Rodriguez PC, et al. J. Exp. Med 2005;202:931-939. [PubMed: 16186186]

9. Sinha P, Clements VK, Fulton AM, Ostrand-Rosenberg S. Cancer Res 2007;67:4507-4513. [PubMed: 17483367]

10. Sinha P, et al. J. Immunol 2008;181:4666-4675. [PubMed: 18802069]

11. Cheng P, et al. J. Exp. Med 2008;205:2235-2249. [PubMed: 18809714]

12. Gorter A, Meri S. Immunol. Today 1999;20:576-582. [PubMed: 10562709]

Nat Biotechnol. Author manuscript; available in PMC 2009 December 31. 


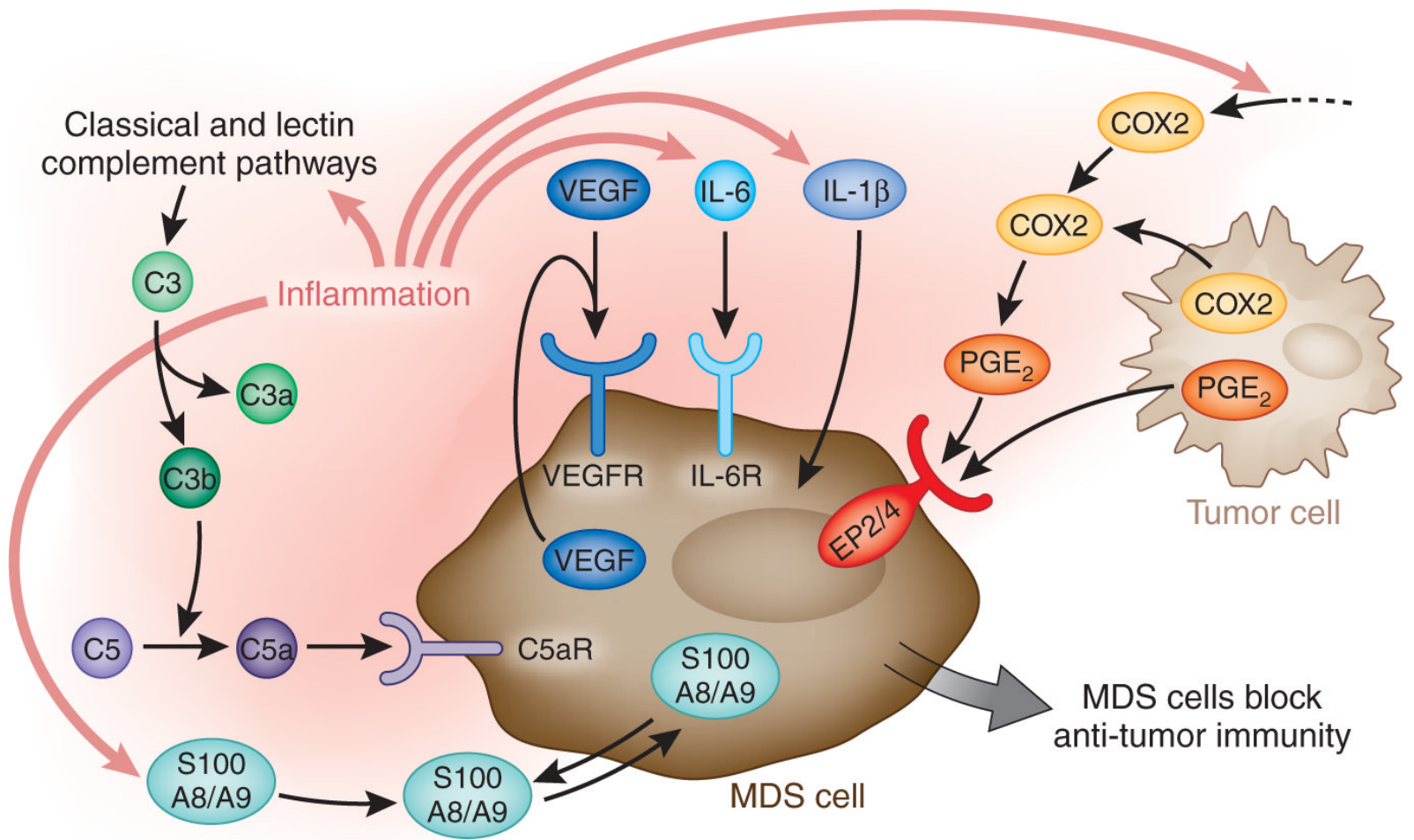

Figure 1.

Multiple pro-inflammatory mediators in the tumor microenvironment increase the accumulation of MDS cells and enhance their immunosuppressive activity. MDS cell accumulation is driven by the proinflammatory factors prostaglandin E2 ( $\left.\mathrm{PGE}_{2}\right), \mathrm{S} 100 \mathrm{~A} 8 / \mathrm{A} 9$ proteins, vascular endothelial growth factor (VEGF), cytokines IL- $1 \beta$ and IL-6, and complement component $\mathrm{C} 5 \mathrm{a}$ (ref. 1), all of which are produced in the tumor microenvironment by host stromal cells. COX2 and $\mathrm{PGE}_{2}$ can also be secreted by tumor cells. MDS cells express receptors for all of these molecules except IL-1 $\beta$. Besides increasing MDS cell abundance and potency, C5a and S100A8/A9 proteins are chemotactic, attracting MDS cells to tumor sites. VEGF and S100A8/A9 proteins are produced by both MDS and other host cells, providing an autocrine regulatory pathway for maintaining MDS cell levels. By activating MDS cells, these pro-inflammatory mediators inhibit anti-tumor immunity and interfere with cell-mediated immunotherapies. 